\title{
Fast outflows of neutral hydrogen in radio galaxies
}

\author{
T. A. Oosterloo ${ }^{1}$, R. Morganti ${ }^{1}$, B. H. C. Emonts ${ }^{2}$ \\ and C. N. Tadhunter ${ }^{3}$
}

${ }^{1}$ Netherlands Foundation for Research in Astronomy, Postbus 2, NL-7990 AA, Dwingeloo, NL email: oosterloo@astron.nl

${ }^{2}$ Kapteyn Astronomical Institute, RuG, Landleven 12, 9747 AD, Groningen, NL

${ }^{3}$ Dept. Physics \& Astronomy, University of Sheffield, S7 3RH, UK

\begin{abstract}
AGN activity is known to drive fast outflows of gas. We report the discovery of fast outflows of neutral gas with velocities over $1000 \mathrm{~km} / \mathrm{s}$ in a number of radio galaxies. In the best studied object, 3C 293, the kinematical properties of the neutral and ionised outflows are similar, indicating a common origin. Moreover, the outflow appears to be located near the radio lobes and not near the nucleus. This suggests that the interaction between the radio jet and the ISM is driving the outflow.
\end{abstract}

\section{Discovery of fast $\mathrm{HI}$ outflows}

AGN activity is increasingly recognised to play an important role in the evolution of galaxies. Particularly important in this respect are the gas outflows that can be generated by the nuclear activity, and the significant effect that these outflows can have (together with the outflows generated by starbursts) on the interstellar medium (ISM) of a galaxy and hence on its evolution. It is, therefore, particularly interesting that in a number of radio galaxies we have now discovered evidence for fast outflows of neutral gas having velocities larger than $1000 \mathrm{~km} / \mathrm{s}$. This shows that, despite the highly energetic phenomena that must be associated with these fast outflows, part of the gas remains, or becomes again, neutral. The discovery of fast outflows of neutral hydrogen in radio galaxies was done using H I observations performed with the Westerbork Synthesis Radio Telescope (WSRT), taking advantage of the new $20 \mathrm{MHz}$ broad-band system and of its excellent bandpass stability. The first fast H I outflow was found in the radio galaxy 3C 293 (Fig. 1, Morganti et al. 2003), but observations carried out recently (again using the WSRT) have revealed fast $\mathrm{H}$ I outflows in five objects (of the 10 observed) with line widths ranging from 800 up to $2000 \mathrm{~km} / \mathrm{s}$.

The optical depths of the broad, shallow absorption are low (typically around 0.001). Nevertheless, due to the large velocity width, the implied column densities are high: around $10^{21} \mathrm{~cm}^{-2}$ for a $T_{\text {spin }}$ of a few hundred $\mathrm{K}$. The observed objects were selected to have a young stellar population component. Such a young stellar population might indicate the presence of significant amounts of gas in the galaxy. A further important characteristic of the galaxies with fast gaseous outflows is that the radio activity started (or re-started) very recently in these galaxies ( $\lesssim 10^{6}$ years ago).

Gas outflows are also detected in quasars and Seyfert galaxies. For quasars, Elvis (2000) suggested they are connected with regions close to the accretion-disk. In Seyferts, radiation and/or wind pressure may drive the outflows (Dopita et al. 2002) although in radio-loud Seyferts they could be due to jet-cloud interactions (e.g. Oosterloo et al. 2000). Starburst winds are detected in a number of nearby galaxies (Heckman et al. 1990). 


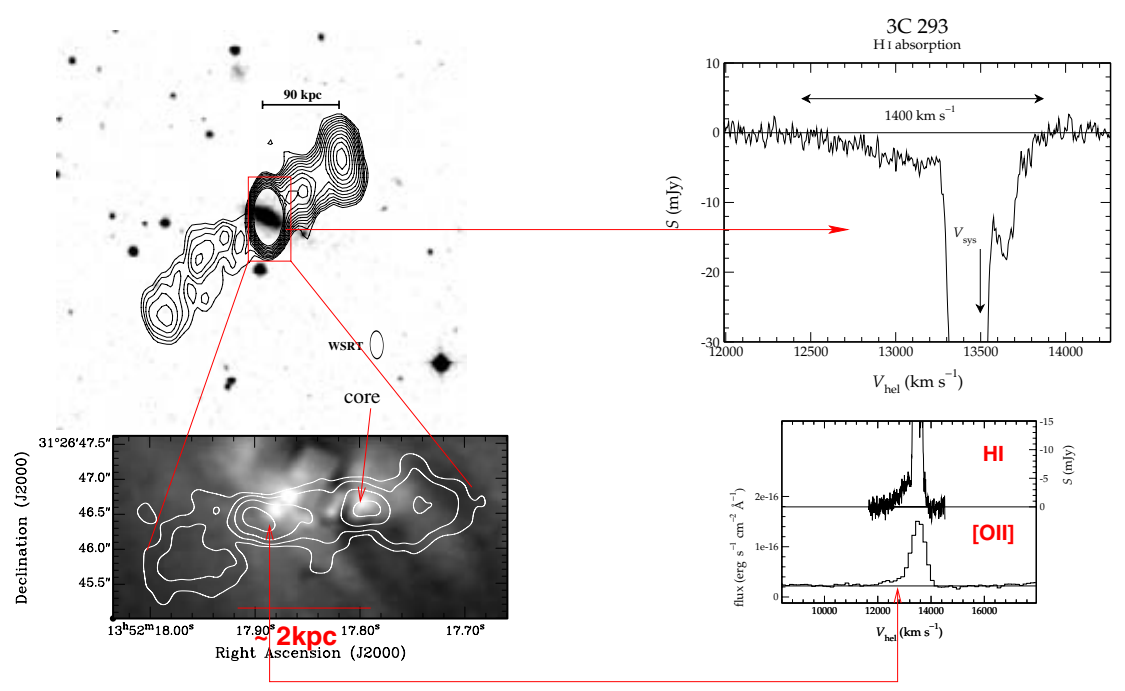

Figure 1. The data for the nearby radio galaxy 3C 293. Top left: the large scale radio continuum. Bottom left: High resolution radio contours of the central region (MERLIN observations from Beswick et al. 2002) superimposed to an HST image. Top right: zoom-in of the WSRT H I absorption profile, where the broad, shallow absorption is clearly visible (Morganti et al. 2003). Bottom right: comparison between the H I absorption and the [O II] $3727 \AA$ profile. The similarity of the broad, blueshifted wing in the two profiles is evident. The broad wing in the optical spectrum is detected at the position of the E radio lobe, not near the core of the radio galaxy.

\section{The case of the nearby radio galaxy $3 \mathrm{C} 293$}

A key observation to discriminate between the mechanisms responsible for gas outflows is to identify the location where the outflow (both in ionised and neutral gas) is occurring. For 3C 293, new optical spectra (Emonts et al. in prep.) show that the optical emission lines also contain a broad blueshifted component that is very similar in width to the broad $\mathrm{H} \mathrm{I}$ absorption. This optical outflow is detected at the position of the eastern radio lobe, 1 kpc from the nucleus (see Fig. 1). The similarity in the spectra suggests that the H I and ionised gas outflows have a common origin. The fact that the broad optical outflow is not detected near the nucleus but near a radio lobe, may imply that the interaction between the radio plasma and the ISM is responsible for the outflow. A possible scenario is that the radio jet hits molecular or neutral clouds in the ISM. These clouds fragment and are accelerated and ionised by the jet shocks. Due to efficient cooling, dense fragments will quickly recombine to form dense, fast moving clouds of neutral (and molecular) gas. Mellema et al. (2002) proposed such a mechanism to explain the phenomenon of jet-induced star formation in (mostly, but not only) high-z radio galaxies.

\section{References}

Beswick, A. J., Pedlar, A., \& Holloway, A. J. 2002, MNRAS, 369, 620

Dopita, M. A., et al. 2002, ApJ, 572, 753

Elvis, M. 2000, ApJ, 545, 63

Evans, A. S., Sanders, D. B., Surace, J. A., \& Mazzarella, J. M. 1999, ApJ, 511, 730

Heckman, T. M., Armus, L., \& Miley, G. K. 1990, ApJS, 74, 833

Mellema, G. Kurk, J. D., \& Röttgering, H. 2002, A\&A, 395, L1

Morganti, R., Oosterloo, T., Emonts, B., van der Hulst, J. M., \& Tadhunter, C. 2003, ApJ, 593, L69

Oosterloo, T., et al. 2000, AJ, 119, 2085 